\title{
JUURNAL.RU
}

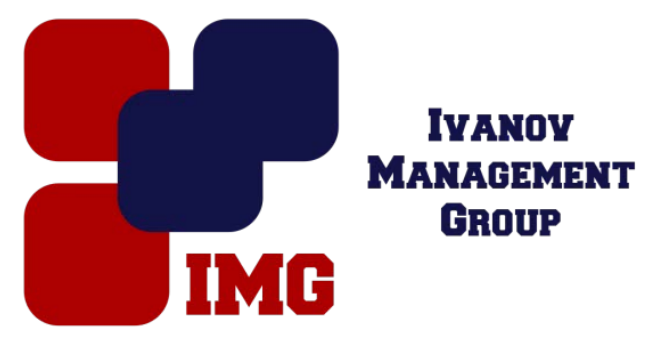

Литвиненкова 3.Н., Осиюк Е.А. Санкт-Петербургский государственный университет гражданской авиаиии Санкт-Петербург, Россия

doi: 10.18411/lj-28-02-2017-1-08

idsp 000001:lj-28-02-2017-1-08

\section{Влияние на прочность местной потери устойчивости в зоне трещины- разреза в тонкой растянутой пластине}

\section{Аннотация}

В конструкциях большинства летательных аппаратов есть тонкостенные элементы (обшивка самолета) с технологическими и эксплуатационными дефектами типа остроконечных надрезов, трещин и иных концентраторов напряжений.

Принцип безопасного повреждения, применяемый в наше время наряду с принципом безопасного ресурса, допускает наличие подобных дефектов, но ставит задачу перед конструкторами учитывать их влияние на разрушающую нагрузку при расчете прочностного ресурса воздушного судна.

В работе дается уточненное решение задачи о растянутой нормальными напряжениями пластине с трещиной-разрезом с учетом депланации (выпучивания) в зоне трещины. При расчете напряженно-деформированного состояния используются соотношения двумерной теории упругости в комплексной форме. Для оценки влияния выпучивания пластины на ее прочность применяется обобщенный метод Бубнова-Галеркина и энергетический подход Гриффитса.

Установлено, что в момент потери устойчивости предельная нагрузка резко снижается (на 25\%), а при дальнейшем росте зоны выпучивания меняется незначительно. Этот эффект существенно сказывается на прочности пластинчатых элементов конструкций летательных аппаратов и должен учитываться на этапе проектирования конструкций.

Ключевые слова: прочность, обшивка самолета, пластина, трещина-разрез, концентрация напряжений, выпучивание (депланация), разрушающая нагрузка, безопасное повреждение.

\section{Abstract}

The paper gives a refined solution of the problem concerning extended by the normal strain plate that has a crack - cut with consideration of warping (buckling) in the crack zone. When calculating strain-deformed state the ratio of the two-dimensional theory of elasticity is used. To evaluate the influence of buckling of the plate on its 
strength the generalized method of Bubnov-Galerkin and Griffith energy approach is applied.

Keywords:strength, aircraft covering, plate, crack - the cut, the concentration of stress, buckling (warping), breaking load, safe damage.

\section{Введение}

Современная обшивка самолетов состоит из панелей или отдельных листов из алюминиевых сплавов (или титана и нержавеющей стали), отформованных по поверхности крыльев или фюзеляжа. В наше время в основном применяется жесткая металлическая обшивка. Она воспринимает нагрузки в виде крутящих и изгибающих моментов, внешние аэродинамические нагрузки и нагрузки перерезывающих сил, воздействующих на каркас самолета. Для производства работающей обшивки применяют титановые, алюминиевые и стальные сплавы, композиционные материалы. В конструкциях сверхзвуковых самолетов чаще всего встречаются титан и сталь.

При обеспечении безопасности полетов на современном этапе наряду с принципом «безопасного ресурса» используется также принцип «безопасного повреждения» [1], который предусматривает наличие в элементах конструкций начальных металлургических и технологических дефектов и образование в них трещин по мере накопления эксплуатационных повреждений.

Опыт эксплуатации и ремонта летательных аппаратов показывает, что задолго до разрушения всей конструкции появляются дефекты ее элементов, такие как трещины обшивки, местная потеря устойчивости обшивки (выпучивание), выпадение заклепок и т.д.

Понятие «безопасных повреждений» относится к конструкции, спроектированной таким образом, чтобы минимизировать возможность выхода летательного аппарата из строя из-за распространения невыявленных дефектовтрещин или других повреждений.

Таким образом, прочностной ресурс воздушного судна следует определять на основе критерия прочности, учитывающего наличие дефектов в материале конструкции и развитие трещин, которые не приводили бы к катастрофическому исходу.

Определение напряженно-деформированного состояния элементов конструкций, содержащих концентраторы напряжений типа трещин, является наиболее ответственным и сложным этапом расчета на прочность. Известно, что напряженно-деформированное состояние тела с трещиной полностью характеризуется величиной коэффициента интенсивности напряжений (КИН). На понятии КИН основаны практически все известные в настоящее время критерии хрупкого и квази-хрупкого разрушения, а также зависимости, описывающие рост усталостных трещин. Кроме того, рост трещины в тонколистовом материале сопровождается локальным выпучиванием в зоне трещины, оказывающем влияние на процесс разрушения [2, 3, 4].

В этой связи актуальным представляется изучение распределения напряжений в окрестности одиночных дефектов в пластинах с использованием разрешающих уравнений уточненных классических теорий и последующее построение критериев предельного равновесия с позиций теории разрушения.

\section{Постановка и решение задачи}

В данной работе в качестве базовой рассматривается задача о растяжении 
плоскости с прямолинейной трещиной - разрезом длиной 2a

$$
\begin{gathered}
-\mathrm{a} \leq \mathrm{x}_{1}^{0} \leq \mathrm{a} \\
\mathrm{x}_{2}^{0} \geq 0,
\end{gathered}
$$

с берегами, свободными от напряжений.

При введении локальной полярной системы координат с полюсом в конце разреза $\rho, \varphi$, получена стандартная асимптотика напряжений и смещений (для напряжений - корневая особенность $\sqrt{a /(2 \rho)})$.

Подробно анализируется явление локальной потери устойчивости частей пластины, примыкающих к разрезу.

Местное выпучивание возникает в растянутой нормальными напряжениями $\sigma_{y}^{\infty}=P$ пластине в связи с наличием зоны сжимающих напряжений в области трещины, величина которых устанавливается при исследовании докритического состояния пластины по методу Вестергард.

Уравнения Вестергард имют вид [5]:

$$
\left\{\begin{array}{c}
\sigma_{11}=\operatorname{Re} \mathrm{Z}-\mathrm{y} \operatorname{Im} \mathrm{Z}^{\prime} \\
\sigma_{22}=\operatorname{Re} \mathrm{Z}+\mathrm{yIm} \mathrm{Z}^{\prime} \\
\tau_{12}=-\mathrm{y} \operatorname{Re} \mathrm{Z}^{\prime}
\end{array}\right.
$$

$\mathrm{Z}=\frac{\text { где }}{\frac{\mathrm{Pz}}{\sqrt{\mathrm{z}^{2}-\mathrm{a}^{2}}}}, \mathrm{z}=\mathrm{x}_{1}^{0}+\mathrm{ix}_{2}^{0}$

Отметим, что функция Вестергард Zсвязана с комплексными потенциалами Колосова-Мусхелишвили [6] следующим образом:

$$
\Phi(\mathrm{z})=\frac{1}{2} Z, \quad \Psi(z)=-\frac{1}{2} z Z^{\prime}
$$

Докритические напряжения в растянутой пластине с разрезом определяются по формулам:

$$
\left\{\begin{array}{c}
\sigma_{\zeta}^{0}=\frac{\rho^{0}}{\sqrt{r^{0}}} \cos \left(\varphi^{0}-\frac{\Psi^{0}}{2}\right)-\frac{\eta}{\sqrt{\left(r^{0}\right)^{3}}} \sin \frac{3}{2} \Psi^{0}-1 \\
\sigma_{\eta}^{0}=\frac{\rho^{0}}{\sqrt{r^{0}}} \cos \left(\varphi^{0}-\frac{\Psi^{0}}{2}\right)+\frac{\eta}{\sqrt{\left(r^{0}\right)^{3}}} \sin \frac{3}{2} \Psi^{0} \\
\tau_{\zeta \eta}^{0}=\frac{\eta}{\sqrt{\left(r^{0}\right)^{3}}} \cos \frac{3}{2} \Psi^{0}
\end{array}\right.
$$

Здесь

$$
\begin{gathered}
\zeta=\frac{x_{1}^{0}}{a} ; \eta=\frac{x_{2}^{0}}{a} ; \quad \rho^{0}=\sqrt{\zeta^{2}+\eta^{2}} ; \\
r^{0}=\sqrt{\left(\zeta^{2}-\eta^{2}-1\right)^{2}+4 \zeta^{2} \eta^{2}} ; \quad \varphi^{0}=\operatorname{arctg} \frac{\pi}{\zeta}
\end{gathered}
$$




$$
\begin{gathered}
\Psi^{0}=\left\{\begin{array}{c}
\operatorname{arctg} \frac{2 \zeta \eta}{\zeta^{2}-\eta^{2}-1}+\pi, \quad \zeta<1 \\
\operatorname{arctg} \frac{2 \zeta \eta}{\zeta^{2}-\eta^{2}-1}, \quad \zeta \geq 1
\end{array}\right. \\
\sigma_{\zeta}^{0}=\frac{\sigma_{11}}{\mathrm{P}} ; \quad \sigma_{\eta}^{0}=\frac{\sigma_{22}}{\mathrm{P}} ; \tau_{\zeta \eta}^{0}=\frac{\tau_{12}}{P}
\end{gathered}
$$

Напряжения $\sigma_{\zeta}^{0}, \sigma_{\eta}^{0}, \tau_{\zeta \eta}^{0}$ были подсчитаны на ЭВМ на прямых $\mathrm{x}= \pm a$ при различных значениях $\eta$ от 0,01 до 2,5 . Анализ полученных результатов позволяет сделать вывод, что сжимающие напряжения на кромках $\mathrm{x}= \pm a$ максимальны при $0 \leq \eta<0,5$ и близки к 0 при $\eta \geq 1,1$.

Критические (эйлеровы) напряжения определяются из дифференциального уравнения задачи:

Здесь

$$
\mathrm{D}^{4} \omega-\mathrm{Pha}^{2}\left(\sigma_{\zeta}^{0} \frac{\partial^{2} \tilde{\omega}}{\partial \zeta^{2}}+\sigma_{\eta}^{0} \frac{\partial^{2} \varpi}{\partial \eta^{2}}+2 \tau_{\zeta \eta}^{0} \frac{\partial^{2} \varpi}{\partial \zeta \partial \eta}\right)=0
$$

$$
\mathrm{D}=\frac{\mathrm{Eh}^{3}}{12\left(1-v^{2}\right)}
$$

h -толщина пластины.

Зона локального выпучивания моделируется полосой шириной $2 a$, нагруженной по длинным кромкам напряжениями, полученными по формулам (2).

На длинных кромках полосы приняты условия упругого защемления с коэффициентом упругой заделки $\beta$. Это связано с тем, что при небольшом прогибе пластина (полоса) работает в условиях жесткого защемления длинных сторон ее контура, а при прогибе, соизмеримом с толщиной пластины, ее края можно считать с достаточной степенью точности шарнирно опертыми.

В дальнейшем проводится численный эксперимент для двух крайних случаев:

a) жесткой заделки (при $\beta=\infty)$;

б) шарнирного опирания (при $\beta=0$ )

краев полосы, то есть при $\zeta= \pm 1$ выполняются граничные условия:

$$
\left\{\begin{array}{c}
\text { a) } \tilde{\omega}=0, \quad \frac{\partial \omega}{\partial \zeta}=0 \text { при } \zeta= \pm 1 \\
\text { б) } \widetilde{\omega}=0, \quad \frac{\partial^{2} \omega}{\partial \eta^{2}}+v \frac{\partial^{2} \varpi}{\partial \zeta^{2}}=0 \text { при } \zeta= \pm 1 \\
\text { При } \eta=0(\text { (берег трещины }- \text { разреза) граничные условия таковы: } \\
\frac{\partial^{2} \omega}{\partial \eta^{2}}+v \frac{\partial^{2} \omega}{\partial \zeta^{2}}=0, \quad \frac{\partial^{3} \omega}{\partial \eta^{3}}+(2-v) \frac{\partial^{3} \omega}{\partial \eta \partial \zeta^{2}}=0 \\
\text { При } \eta \rightarrow \infty, \quad \tilde{\omega} \rightarrow 0
\end{array}\right.
$$


Краевая задача (3) -(4) решается вариационным методом (обобщенный метод Бубнова-Галеркина) [7].

$$
\begin{gathered}
\int_{-1}^{1} \int_{0}^{\infty}\left\{\nabla^{4} \omega-\lambda\left[\sigma_{\zeta}^{0} \frac{\partial^{2} \widetilde{\omega}}{\partial \zeta^{2}}+\sigma_{\eta}^{0} \frac{\partial^{2} \varpi}{\partial \eta^{2}}+2 \tau_{\zeta \eta}^{0} \frac{\partial^{2} \varpi}{\partial \zeta \partial \eta}\right]\right\} \delta \omega \mathrm{d} \zeta \mathrm{d} \eta- \\
-\left.\int_{-1}^{1}\left(\frac{\partial^{2} \omega}{\partial \eta^{2}}+v \frac{\partial^{2} \omega}{\partial \zeta^{2}}\right) \cdot \frac{\partial}{\partial \eta} \delta \omega \mathrm{d} \zeta\right|_{\eta=0}-\left.\int_{-1}^{1}\left[\frac{\partial^{3} \omega}{\partial \eta^{3}}+(2-v) \frac{\partial^{3} \omega}{\partial \eta \partial \zeta^{2}}\right] \delta \omega \mathrm{d} \zeta\right|_{\eta=0} \\
=0
\end{gathered}
$$

$\lambda=\frac{\mathrm{Pha}^{2}}{\mathrm{D}}$

Форма выпученной поверхности задается в виде:

$$
\begin{aligned}
& \omega(\zeta, \eta)=\sum_{\mathrm{m}=2}^{\mathrm{p}} \sum_{\mathrm{n}=1}^{\mathrm{q}} \mathrm{A}_{\mathrm{mn}} \omega_{\mathrm{mn}} \\
& \text { где } \omega_{\mathrm{mn}}=\cos ^{\mathrm{m}} \frac{\pi \zeta}{2} \mathrm{e}^{-\mathrm{n \eta}}
\end{aligned}
$$

Подставляя (7) в (5), получим квадратичную форму от параметров $A_{m n}$. Минимизируя ее, находим $r$ значений параметра $\lambda$, наименьшее из которых позволяет определить из выражения (6) критическое значение растягивающей нагрузки.

$$
\mathrm{P}_{\mathrm{\kappa p}}=\frac{\mathrm{D} \lambda_{\min }}{\mathrm{ha}^{2}}=\mathrm{K} \frac{\pi^{2} \mathrm{D}}{\mathrm{a}^{2} \mathrm{~h}}=\mathrm{K}_{1} \mathrm{E}\left(\frac{\mathrm{h}}{\mathrm{a}}\right)^{2}
$$

Численная реализация указанного метода осуществляется на ЭВМ. Были получены следующие значения коэффициента $K_{1}$ в формуле (8):

$$
1,08 \leq K_{1} \leq 1,2
$$

При сравнении полученной в соответствии с формулой (8) зависимости критической нагрузки от толщины для образцов из стали и из алюминиевых сплавов с экспериментальными данными усматривается их хорошее согласование.

Потеря устойчивости плоской пластины приводит к изменению напряженного состояния у вершины трещины и следовательно, влияет на процесс разрушения.

Далее в работе оценивается прочность пластины с выпучиванием в зоне трещины. При этом предполагается, что линии главных напряжений обтекают выпученную область таким же образом, как если бы на ее месте в пластине имелось сквозное эллиптическое отверстие с полуосями $\max |x|=a$ и $\max |y|=$ $b, \quad b=1,1 a$.

Основное уравнение теории Гриффитса имеет вид [8]:

$$
\frac{\partial}{\partial \mathrm{a}}[\mathrm{T}(\mathrm{a})-\mathrm{W}(\mathrm{a}, \mathrm{b}, \mathrm{P})=0],
$$

где $T(a)=4 \gamma_{0} a-$ поверхностная энергия трещины, 
$\gamma_{0}-$ плотность поверхностной энергии материала,

$W(a, b, P)-$ энергия упругого деформирования, обусловленная раскрытием трещины и увеличением протяженности выпученной зоны при возрастании напряжений Р.

Для $W(a, b, P)$ имеем:

$$
W(a, b, P)=\frac{\pi P^{2}}{2 E}\left(2 a^{2}+a b\right)
$$

Подставляя выражения для $\mathrm{T}$ и $\mathrm{W}$ в уравнение Гриффитса, находим разрушающую нагрузку $P^{*}$ (с учетом выпучивания).

$$
\mathrm{P}^{*}=\sqrt{\frac{8 \gamma E}{\pi(4 a+b)}}
$$

Обозначим разрушающую нагрузку невыпученной пластины с трещиной через $\mathrm{P}_{0}^{*}$. Эта величина может быть получена по формуле для $\mathrm{P}^{*}$ при $\mathrm{b}=0$

$$
\mathrm{P}_{0}^{*}=\sqrt{\frac{2 \gamma \mathrm{E}}{\mathrm{a} \pi}}
$$

которая совпадает с известной формулой Ирвина.

Рассмотрим отношение

$$
\zeta=\frac{\mathrm{P}^{*}}{\mathrm{P}_{0}^{*}}=\frac{2}{\sqrt{4+\mathrm{b} / \mathrm{a}}}
$$

Предположим, что нагрузка, приложенная к бесконечной пластине, превышает критическую:

$$
\mathrm{P}=\mathrm{KE}\left(\frac{\mathrm{h}}{\mathrm{a}}\right)^{2},
$$

гдеК > 1,08.

Оценим влияние выпучивания на разрушающую нагрузку, установив зависимость параметра $\zeta$ от коэффициента внешней нагрузки K:

$$
\zeta=f(K)
$$

Анализ этой зависимости приводит к выводу, что в момент потери устойчивости предельная нагрузка резко падает $\approx$ на $25 \%$, а при дальнейшем росте зоны выпучивания меняется незначительно.

\section{Заключение}

Полученный в работе результат свидетельствует о том, что депланация пластинчатых элементов конструкций летательных аппаратов в зоне трещины приводит к понижению разрушающей нагрузки на 20-25\%. Этот фактор должен приниматься во внимание конструкторами для обеспечения безопасности полетов. 


\section{Литература}

1. Нестеренко Г.И. Требования по усталости и живучести конструкций гражданских самолетов.// Научный вестник МГТУГА № 163, 2011

2. Гольцев В.Ю. и др. Заводская лаборатория № 1, 1961

3. Литвиненкова 3.Н.Об устойчивости растянутой пластины с внутренней трещиной.// Известия АН СССР, Механика твердого тела № 5, 1973

4. Литвиненкова 3.Н.Разрушающая нагрузка растянутых плоских элементов конструкций самолета (обшивки) у мест выточек.// Вестник СПбГУГА, выпуск 2, 2011

5. Вестергард Г.М.Несущие давления и трещины.// Журнал прикладной механики, том 6, с. А 49-53, 1939

6. Мусхелишвили И.Н.Некоторые основные задачи математической теории упругости. М., «Наука», 1966.

7. Галеркин Б.Г.Собрание сочинений. Т. 1.// Изд-во АН СССР, 1952

8. Griffith A.A. The theory of rupture.// Proc. First. Inter. Congress Appl. Mech. Delft., p. 55-63, 1924 